\title{
Kinematics and center of axial rotation during walking after medial pivot type total knee arthroplasty
}

Kota Miura ${ }^{1 *} \mathbb{D}$, Yasumitsu Ohkoshi ${ }^{2}$, Takumi Ino ${ }^{1,3}$, Kengo Ukishiro ${ }^{1}$, Kensaku Kawakami $^{4}$, Sho'ji Suzuki ${ }^{5}$, Ko Suzuki ${ }^{2}$ and Tatsunori Maeda ${ }^{2}$

\begin{abstract}
Purpose: In recent years, the medial pivot (MP) type total knee arthroplasty (TKA) implant has been developed and marketed for achieving more natural kinematics with MP. However, little is known about the pivot pattern during walking after MP type TKA. This study aimed to determine the kinematics and center of axial rotation during walking after MP type TKA.

Methods: This randomized prospective study enrolled 40 patients with MP type TKA, 20 with cruciate-substituting TKA (MP-CS group), 20 with posterior-stabilized TKA (MP-PS group), and 10 healthy volunteers (control group). The kinematics and center of axial rotation during overground walking were measured by a three-dimensional motion analysis system. The six-degrees-of-freedom kinematics of the knee were calculated by the point cluster method.

Results: The amount of change in knee flexion in early stance phase was significantly lower in the MP-CS and MPPS groups than in the control group. The femur showed anterior translation during early stance phase in all three groups. The median center of axial rotation in the transverse plane was predominantly on the lateral side of the knee during stance in all groups.

Conclusions: Kinematics during gait are thought to be determined by physical posture, the kinetic chain during weight-bearing, and the kinematic features of adjacent structures, such as the behavior of the biarticular muscles. MP-CS and MP-PS did not necessarily induce rotational motion centered on the medial ball-in-socket component during walking; translational and lateral pivoting movements were also observed. Long-term follow-up is needed to monitor for polyethylene wear and implant loosening.
\end{abstract}

Keywords: Lateral pivot, Total knee arthroplasty, Center of axial rotation, Kinematics, Walking

\section{Background}

Six-degrees-of-freedom (6DOF) kinematics of the knee joint during walking are determined by physical posture, the position of the body's barycenter, the kinetic chain during weight-bearing, muscle coordination and antagonism, and the kinematic features of adjacent structures, including the behavior of the biarticular muscles $[2,8$, $12,15]$. In recent years, many types of implants for total

\footnotetext{
* Correspondence: kouta@hakodate-seikei.com

'Department of Rehabilitation, Hakodate Orthopedics Clinic, 2-115,

Ishikawa-cho, Hakodate-shi, Hokkaido 041-0802, Japan

Full list of author information is available at the end of the article
}

knee arthroplasty (TKA) have become available. The center of axial rotation (COR) of the knee joint is expressed as either medial or lateral pivot depending on the position of the COR on the tibial plateau [3]. Medial pivot (MP) type TKA is designed to create a medial ballin-socket structure to ensure a medial location of the COR for medial pivot motion. Several motion analysis studies [4, 21-23] have been reported for MP type TKA; however, the 6DOF kinematic features of the knee joint when overground walking after surgery have yet to be fully elucidated. Moreover, the question of whether medial pivot motion is physiological remains controversial.
Springer Open
๑ The Author(s). 2020 Open Access This article is licensed under a Creative Commons Attribution 4.0 International License, which permits use, sharing, adaptation, distribution and reproduction in any medium or format, as long as you give appropriate credit to the original author(s) and the source, provide a link to the Creative Commons licence, and indicate if changes were made. The images or other third party material in this article are included in the article's Creative Commons licence, unless indicated otherwise in a credit line to the material. If material is not included in the article's Creative Commons licence and your intended use is not permitted by statutory regulation or exceeds the permitted use, you will need to obtain permission directly from the copyright holder. To view a copy of this licence, visit http://creativecommons.org/licenses/by/4.0/. 
Several fluoroscopic studies $[6,7,10,13,15]$ of walking and lunge motions in both living human knees and cadaveric knees show medial pivot motion at the knee. By contrast, a fluoroscopic study by Kozanek et al. [17] found that lateral pivot occurred during walking in healthy subjects. Furthermore, Koo et al. [16] analyzed walking in healthy subjects by using an optical motion capture technique and reported the presence of lateral pivot motion. The findings of these reports are inconsistent with the position of the COR, even in healthy knees. The present study aimed to determine the 6DOF knee kinematics and location of the COR in the transverse plane of the knee during walking in patients with MP type TKA.

\section{Methods}

Patients who underwent TKA using the Evolution ${ }^{\circ}$ Medial Pivot Knee System (MicroPort Orthopedics Inc., Arlington, VA) at our hospital from February 2015 to February 2016 were randomly allocated (1:1) using sequentially numbered, opaque, sealed envelopes to a cruciate-substituting (MP-CS) group or a posteriorstabilized (MP-PS) group in a prospective manner. The inclusion criteria were 1 ) age 20 years or older; 2) osteoarthritis (OA) of the knee with a Kellgren-Lawrence grade of $\geq 2$; and 3 ) a femorotibial angle (FTA) of $<190^{\circ}$ on a radiographic frontal view in the standing posture (range of FTA, $172.0^{\circ}-189.9^{\circ}$ ). Patients with rheumatoid arthritis, lateral type $\mathrm{OA}$ with valgus alignment, or a limp were excluded. Applying these criteria, 20 knees of 20 patients undergoing MP-CS TKA ( 1 male, 19 female) and 20 knees of 20 patients undergoing MP-PS TKA (2 male, 18 female) were included in the study. Two patients in whom a postoperative evaluation was not possible (1 with lumbar disease and 1 with traumatic patellar tendon rupture following MP-CS TKA) were subsequently excluded. Two further patients ( 1 in the MP-CS group and 1 in the MP-PS group) could not attend the postoperative evaluation for social reasons. Clinical evaluations were performed before and 1 year after surgery. The motion analyses were performed 1 year after surgery in 17 knees of 17 patients ( 1 male, 16 female, mean age $71.6 \pm 5.5$ years, body mass index [BMI] 27.4 \pm 4.9$)$ in the MP-CS group and in 19 knees of 19 patients ( 2 male, 17 female, mean age $73.1 \pm 5.5$, BMI $26.8 \pm 3.0$ ) in the MP-PS group. The control group comprised 10 knees of 10 healthy volunteers (4 male, 6 female, mean age $27.8 \pm 4.7$ years, BMI $20.0 \pm 2.0$ ). The clinical items evaluated were range of motion (ROM) and plain standing radiographs. The 2011 Knee Society Score (KSS 2011) was recorded. The $\alpha, \beta, \gamma$, and $\delta$ angles, the FTA, and the WBL ratio were also measured. The medial and lateral joint space widths were measured on varus and valgus stress radiographs using a Telos SE stress device (Telos GmbH, Hungen, Germany). After preoperative planning, surgery was performed by two experienced orthopedists using the same measured resection technique. In all cases, patella replacement and posterior cruciate ligament resection were performed. No medial release was performed and only medial bony spurs were resected. Three-dimensional (3D) motion analysis of walking was performed in all three groups. The 3D motion analysis system consisted of 8 infrared cameras (ProReflex, Qualisys AB Inc., Gothenburg, Sweden) and 2 force plates (OR6; Advanced \% Technology Inc., Watertown, MA). The measurement frequency of the infrared cameras and force plates was set at 120 Hz. To avoid mutual interference, the force plates were arranged independently of each other in the halfway zone of the 8-m walking pathway such that subjects could not recognize the sites of the plates. Each subject walked barefoot on the pathway with a steady gait at a self-selected comfortable walking speed. Measurement was considered complete upon achieving three successful walking cycles. To avoid the influence of fatigue, each subject rested adequately between cycles. Skin markers were attached to the lower limbs (56 markers) and to the acromion on both sides by a physical therapist with detailed knowledge of the surface anatomy of the knee using the point cluster (PC) method [2, 12]. The Qualisys Track Manager 3D motion analysis software program was used to analyze the recorded data by the PC method. One walking cycle was defined as the interval between heel strike of a foot to the next heel strike of the same foot, standardized as $100 \%$, based on information from the infrared cameras synchronized with the floor reaction force sensors. The joint coordinate system for the PC method was set based on the definition of Grood and Suntay [11]. In the tibial coordinate system, the mediolateral axis of the tibia ( $\mathrm{x}$ axis) was defined as a line segment connecting the points several millimeters proximal to the apices of the medial and lateral condyles of the tibia (i.e., points consistent with the articular surface). The line segment on the articular surface passing through the midpoint of the aforementioned line segment and perpendicular to the $\mathrm{x}$ axis was defined as the anteroposterior axis ( $y$ axis). The intersection of the $x$ and $y$ axes was regarded as the origin, and a line passing through the origin and orthogonal to the $x-y$ plane was regarded as the $\mathrm{z}$ axis. In the femoral coordinate system, the line segment connecting the medial and lateral epicondyles of the femur was defined as the transepicondylar axis (TEA), and the midpoint of the TEA was regarded as the origin. The line passing through the origin and parallel to the long axis of the femur was defined as the supra-inferior axis, and the line passing through the origin and perpendicular to the TEA and the suprainferior axis was defined as the anteroposterior axis. The 
kinematics of the knee during walking, shown as movement of the femur with respect to the tibia, were then analyzed. The flexion and extension angles of the knee joint and the rotation and anteroposterior translation movement of the femur were examined. The knee kinematics were analyzed by setting the angle and location of the standing position at rest with the knee fully extended as point zero. The projected transepicondylar axis (pTEA) of the femur in the tibial x-y plane was defined as the pTEA. The change in pTEA after knee motion was defined as the pTEA'. The COR was defined as the intersection of pTEA and pTEA' (Fig. 1). The instantaneous COR (ICOR) was calculated per $0.0083 \mathrm{~s}$ during walking, and all $\mathrm{x}$ coordinates of the ICOR from three trials were assessed in histograms.

The unpaired $t$-test was used to compare the clinical findings between the MP-CS and MP-PS groups. The Mann-Whitney $U$ test was used to compare the postoperative KSS 2011 results between the two groups. The significance level was set at less than 5\%. The KruskalWallis test was used to compare peak values and amounts of change in the knee kinematics and the location of median COR among the three groups with the Dunn-Bonferroni method used for post hoc testing, with the significance level set at $P<0.017$.

The ethics committee of our institution approved this study, and all subjects provided written informed consent after receiving a detailed explanation of the study.

\section{Results}

There was no significant difference in preoperative knee flexion angle, bone morphology angle, or clinical outcome between the MP-CS and MP-PS groups (Table 1). The walking speed was $1.4 \mathrm{~m} / \mathrm{s}$ in the control group. Walking speeds were significantly lower in the MP-CS and MP-PS groups both pre- and postoperatively compared with the control group (Table 2). There was no significant difference in postoperative ROM between the
MP-CS group and the MP-PS group $\left(130.3 \pm 7.0^{\circ}\right.$ vs $\left.133.4 \pm 6.7^{\circ}\right)$. Furthermore, there were no significant differences in radiographic alignment or findings on stress radiography between these two groups (Table 3). The KSS 2011 scores were significantly better in the MP-CS group than in the MP-PS group in terms of patient satisfaction with current knee pain of major category II both overall and for the following specific items: 1 , sitting in a chair; 3, getting out of bed; 4, light housework; and 5, going out or other recreational activity. There were no significant differences in any of the other items (Table 4).

All three groups showed knee flexion twice during one walking cycle and the femur rotated externally from early to mid-stance and then rotated internally (Fig. 2a, b). In the control group, the femur showed anterior translation during early stance phase, followed by maintenance of the anterior position or slight posterior translation, then another anterior translation, and finally posterior translation during late stance phase to swing phase. However, in both the MP-CS and the MP-PS groups, the femur showed anterior translation during early stance phase, maintained the anterior position during mid-stance phase, and showed posterior translation during late stance phase to swing phase (Fig. 2c).

Quantitative analysis of the knee kinematics during walking revealed that the amount of change in knee flexion in early stance phase was significantly lower in the MP-CS and MP-PS groups than in the control group $\left(9.6^{\circ}\right.$ and $12.1^{\circ}$, respectively, vs $\left.17.0^{\circ}\right)$. The femur in stance phase was externally rotated in all three groups, reaching its peak almost simultaneously with peak extension of the knee. The mean angle of maximum external rotation was $4.7^{\circ}$ in the MP-CS group, $4.1^{\circ}$ in the MP-PS group, and $6.9^{\circ}$ in the control group, with a significant difference between the MP-PS group and the control group. The amount of anterior translation of the femur in stance phase was $8.0 \mathrm{~mm}$ in the MP-CS group,
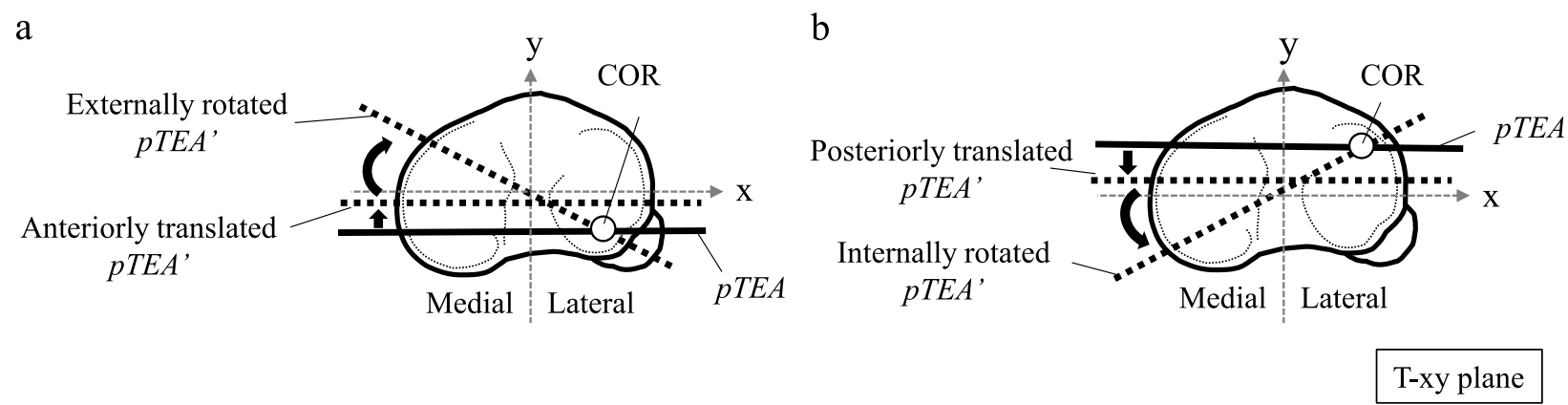

Fig. 1 COR estimation during walking. The projected transepicondylar axis of the femur on the tibial $x$-y plane was defined as the pTEA (solid line). The pTEA' (dotted line) was defined as the change in pTEA after knee motion. The COR was defined as the intersection of pTEA and pTEA'. When the PTEA translated anteriorly and rotated externally, the COR was lateral, that is, lateral pivot (a). When the pTEA translated posteriorly and rotated internally, the COR was lateral (b). COR, center of axial rotation 
Table 1 Comparison of preoperative knee flexion angle, bone morphologic angles, and clinical outcome

\begin{tabular}{|c|c|c|c|c|c|c|c|c|c|}
\hline Group & $\begin{array}{l}\text { Knee } \\
\text { flexion }\left({ }^{\circ}\right)\end{array}$ & FTA $\left(^{\circ}\right)$ & $\begin{array}{l}\text { a angle } \\
\left({ }^{\circ}\right)\end{array}$ & $\begin{array}{l}\beta \text { angle } \\
\left({ }^{\circ}\right)\end{array}$ & $\begin{array}{l}Y \text { angle } \\
\left({ }^{\circ}\right)\end{array}$ & $\begin{array}{l}\delta \text { angle } \\
\left({ }^{\circ}\right)\end{array}$ & WBL (\%) & $\begin{array}{l}\text { Medial dilation of the tibiofemoral } \\
\text { joint }(\mathrm{mm})\end{array}$ & $\begin{array}{l}\text { KSS } 2011 \\
\text { (total) }\end{array}$ \\
\hline MP-CS & $132.5 \pm 9.8$ & $183.0 \pm 4.6$ & $98.5 \pm 2.4$ & $84.0 \pm 3.3$ & - & $81.8 \pm 2.9$ & $9.0 \pm 19.3$ & $6.7 \pm 1.0$ & $84.9 \pm 23.2$ \\
\hline MP-PS & $137.5 \pm 10.7$ & $179.8 \pm 4.4$ & $98.9 \pm 1.6$ & $84.5 \pm 2.2$ & - & $80.5 \pm 3.1$ & $21.4 \pm 16.3$ & $7.2 \pm 1.0$ & $82.9 \pm 23.0$ \\
\hline
\end{tabular}

FTA, femorotibial angle; $a$, angle of the distal femoral end; $\beta$, angle of the proximal tibial end; $\gamma$, femoral flexion angle; $\delta$, posterior tibial slope; WBL Weight bearing line; KSS 20112011 Knee Society Score; MP Medial pivot; CS Cruciate-substituting; PS Posterior-stabilized. There was no significant difference between the two MP groups (unpaired $t$-test and Mann-Whitney $U$ test, significance level $<5 \%$ )

$7.3 \mathrm{~mm}$ in the MP-PS group, and $8.8 \mathrm{~mm}$ in the control group, with no significant difference among the groups.

The median CORs $(\mathrm{x}[\mathrm{cm}], \mathrm{y}[\mathrm{cm}])$ in early stance were $(4.3,1.1)$ in the MP-CS group, $(4.6,1.0)$ in the MPPS group, and $(7.1,0.5)$ in the control group. In all groups, the COR was located in the first quadrant (lateral side). The $\mathrm{x}$-coordinate was significantly smaller and more medial in both TKA groups than in the control group. During mid-stance, the median CORs were (0.7, $0.7)$ in the MP-CS group, $(-0.4,0.8)$ in the MP-PS group, and $(3.1,0.4)$ in the control group; the COR in the MP-PS group was located in the medial quadrant. During late stance, the median CORs were $(1.4,0.5)$ in the MP-CS group, $(3.4,0.2)$ in the MP-PS group, and $(0.9,0.6)$ in the control group; the $\mathrm{x}$-coordinates were located on the lateral side in all groups (Fig. 3). Histogram analysis of the ICORs revealed that they were lateral in $72.2 \%$ of subjects in the MP-CS group, in $70.4 \%$ in the MP-PS group, and in $89.4 \%$ in the control group during early stance, $54.4 \%$ in the MP-CS group, $47.9 \%$ in the MP-PS group, and $67.5 \%$ in the control group during mid-stance, and in $58.8 \%$ in the MP-CS group, $68.6 \%$ in the MP-PS group, and $55.7 \%$ in the control group during late stance to pre swing (Fig. 4). Over time, some knees showed lateral pivot only or coexistence of medial and lateral pivot during early stance in all three groups (Fig. 5).

\section{Discussion}

The control group in this study showed external rotation and anterior translation of the femur in stance phase. Lafortune et al. [18] analyzed knee kinematics in healthy subjects by an optical motion capture technique using metal pins with an optical reflection marker inserted into the tibia and femur. They reported that the femur showed external rotation and anterior translation twice during stance. Koo et al. [16] analyzed the kinematics and ICOR of 23 healthy knees using the PC method and reported that all the average ICORs were located laterally due to the femur underwent external rotation and anterior translation in stance phase during walking. Kozanek et al. [17] analyzed movement of the femur in healthy subjects using biplane radiography and magnetic resonance imaging and reported external rotation and anterior translation in early stance phase during walking. The results of these three studies are consistent with those of our study, indicating the validity of our analyses of knee kinematics.

Quantitative analyses of knee flexion and extension during walking showed that the amounts of change in early stance phase and mid-stance phase were significantly lower in the MP-CS and MP-PS groups than in the control group. McClelland et al. [20] compared knee kinematics during comfortable walking between patients 1 year after TKA and 40 healthy subjects matched for age and sex and reported that the knee flexion angles in both stance and swing phases were significantly smaller in the patients who had undergone TKA, suggesting that control by the quadriceps and hip extensor muscles in loading response remained inadequate 1 year after surgery. A similar phenomenon may have occurred in the patients in the MP-CS and MP-PS groups in this study, whose postoperative walking speed was significantly lower than that of subjects in the control group. This slow

Table 2 Comparison of preoperative and postoperative walking speed

\begin{tabular}{llcr}
\hline & Before surgery & After surgery \\
\hline Walking speed $(\mathrm{m} / \mathrm{s})$ & MP-CS group & $0.9 \pm 0.2^{* \dagger}$ & $1.0 \pm 0.1^{* \$}$ \\
& MP-PS group & $1.1 \pm 0.2^{* \dagger}$ & $1.0 \pm 0.2^{* \S}$ \\
& Control group & & $1.4 \pm 0.1$ \\
\hline
\end{tabular}

MP Medial pivot; CS Cruciate substituting; PS Posterior-stabilized

$P<0.05$, Kruskal-Wallis test,

*Significantly slower than in the control group

$t, \S$ not statistically significant 
Table 3 Comparison of knee flexion angle and radiographic measurements at 1 year after surgery

\begin{tabular}{lllllllll}
\hline & $\begin{array}{l}\text { Knee flexion } \\
\left({ }^{\circ}\right)\end{array}$ & FTA $\left(^{\circ}\right)$ & $\begin{array}{l}\boldsymbol{\alpha} \text { angle } \\
\left({ }^{\circ}\right)\end{array}$ & $\begin{array}{l}\boldsymbol{\beta} \text { angle } \\
\left({ }^{\circ}\right)\end{array}$ & $\begin{array}{l}\boldsymbol{\gamma} \text { angle } \\
\left({ }^{\circ}\right)\end{array}$ & $\begin{array}{l}\boldsymbol{\delta} \text { angle } \\
\left({ }^{\circ}\right)\end{array}$ & WBL $(\%)$ & $\begin{array}{l}\text { Medial dilation of the tibiofemoral joint } \\
(\mathbf{m m})\end{array}$ \\
\hline $\begin{array}{l}\text { MP-CS } \\
\text { group }\end{array}$ & $130.3 \pm 7.0$ & $176.3 \pm 2.7$ & $96.5 \pm 1.9$ & $88.5 \pm 1.5$ & $2.1 \pm 1.9$ & $84.7 \pm 2.4$ & $40.0 \pm 7.2$ & $1.4 \pm 0.7$ \\
MP-PS & $133.4 \pm 6.7$ & $175.2 \pm 2.5$ & $97.0 \pm 1.7$ & $88.7 \pm 1.5$ & $3.0 \pm 2.6$ & $84.0 \pm 2.1$ & $40.8 \pm 13.3$ & $1.6 \pm 0.5$ \\
group & & & & & & & & \\
\hline
\end{tabular}

FTA Femorotibial angle; $\alpha$, femoral component angle; $\beta$, tibial component angle; $\gamma$, femoral component flexion angle; $\delta$, tibial posterior slope; WBL Weight bearing line; MP Medial pivot; CS Cruciate-substituting; PS Posterior-stabilized. There was no significant difference between the two MP groups (unpaired $t$-test, significance level $<5 \%)$

walking pace may also have contributed to the small angle of knee flexion.

External rotation and anterior translation of the femur were observed in stance phase during walking in the MP-CS and MP-PS groups. However, in both these groups, anterior translation occurred in early stance phase. It is thought that the breaking action of the tibia causes the femur to slide forward as the knee flexes [17]. Anterior translation of the femur was maintained in mid-stance phase in the MP-CS and MP-PS groups, and the maximum external rotation angle of the femur in stance phase was significantly smaller in the MP-PS groups than that in the control group. This phenomenon resembles the compensatory movement commonly observed in walking by patients with anterior cruciate ligament (ACL) insufficiency [5, 8], which presumably suppresses the anterolateral rotatory instability and anterior displacement of the tibia. Given that knees treated by TKA are also ACL-insufficient, it is possible that similar compensatory movements occurred in the patients in this study. This possibility requires further investigation involving moment analysis.

Whether or not MP always induces medial pivot motion in the ball-in-socket structure of the medial tibiofemoral joint remains an important issue. As mentioned previously, there was anterior translation and simultaneous external rotation of the femur in early stance phase during walking in both the MP-CS and MP-PS groups. Analysis of this motion in terms of COR revealed lateral pivot (Fig. 1-a). The femur showed posterior translation, which indicates roll back, from late stance to swing phase, and internal rotation of the femur was simultaneously present. Simultaneous occurrence of posterior translation and internal rotation of the femur means lateral pivot when analyzed in terms of COR (Fig. 1-b). Furthermore, all three groups included subjects showing lateral pivot only or coexistence of medial and lateral pivot changing over time in location of the ICOR during early stance (Fig. 5). These results suggest that, in both the MP-CS and MP-PS groups, medial pivot was not constantly induced in stance phase during walking, with lateral pivot being present instead. Banks and Hodge reported that PS and cruciate-retaining (CR) implants showed lateral centers of rotation in stance phase during walking [3]. These provides a view of the COR is not located at a single point because COR is mainly determined by knee kinematics, especially translation and rotation, which depends on the type of motion, implant design, and characteristics of individuals. Therefore, COR kinematics vary according to the type and phase of motion and among individuals, and the COR seemed to vary according to these kinematic differences. The ballin-socket structure of the medial joint in the MP-CS knee does not necessarily induce medial pivot motion, and it is thought that translational and lateral pivot motion may occur. In particular, the occurrence of translation and lateral pivot motion in MP-CS indicates the possibility of overriding of the femoral implant on the tibial socket margin. This shows that the ball-in-socket structure of the MP-CS functions as a restraint mechanism in the anteroposterior direction; however, if overriding of the femoral implant occurs, then there is a concern about wear and breakage of the polyethylene material in the long term. A good outcome for MP 10 years after surgery has been reported previously [14, 19]. However, this result may be due to improvements in the quality of polyethylene inserts. Moreover, better conformity of the inserts has resulted in more anteriorposterior translation, higher wear rates, and a greater wear area $[9,24]$. Therefore, further long-term follow-up is required.

Postoperative satisfaction rated using the KSS (2011) was significantly better in the MP-CS group than in the MP-PS group. This was thought to be because the

Table 4 Comparison of satisfaction with each item and the total KSS 2011 at 1 year after surgery

\begin{tabular}{|c|c|c|c|c|c|c|c|}
\hline Type & 1 Sitting in a chair & 2 Lying in bed & 3 Getting out of bed & 4 Light housework & 5 Going out, recreation & II total & KSS 2011 \\
\hline CS group & $6.1 \pm 1.5^{*}$ & $5.5 \pm 1.8$ & $5.6 \pm 1.8^{*}$ & $5.5 \pm 1.5^{*}$ & $5.5 \pm 1.7^{*}$ & $28.4 \pm 7.5^{*}$ & $130.8 \pm 20.9$ \\
\hline PS group & $4.9 \pm 1.5^{*}$ & $4.8 \pm 1.7$ & $4.3 \pm 1.7^{*}$ & $4.2 \pm 1.6^{*}$ & $3.7 \pm 1.7^{*}$ & $22.0 \pm 7.1^{*}$ & $122.3 \pm 25.5$ \\
\hline
\end{tabular}


a

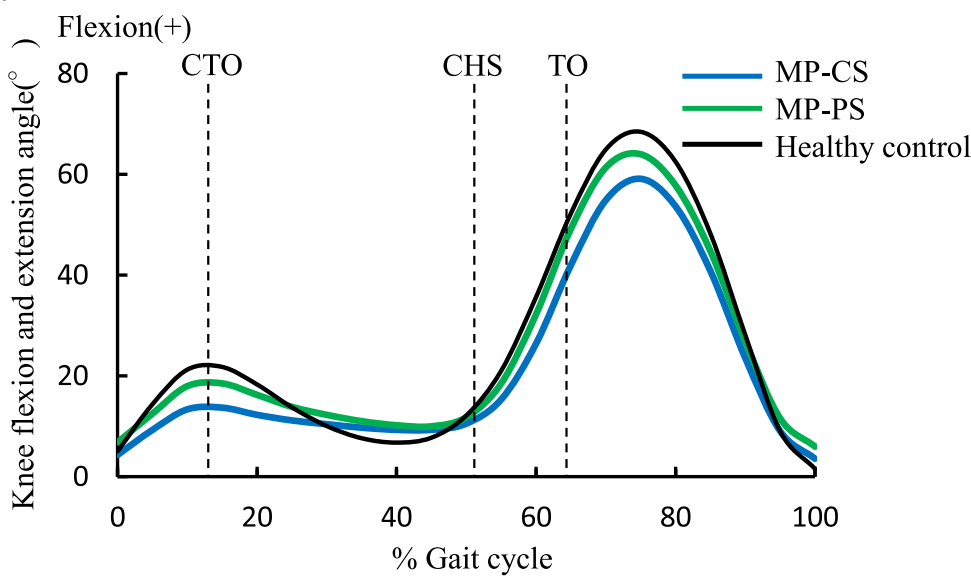

b

Internal rotation $(+)$

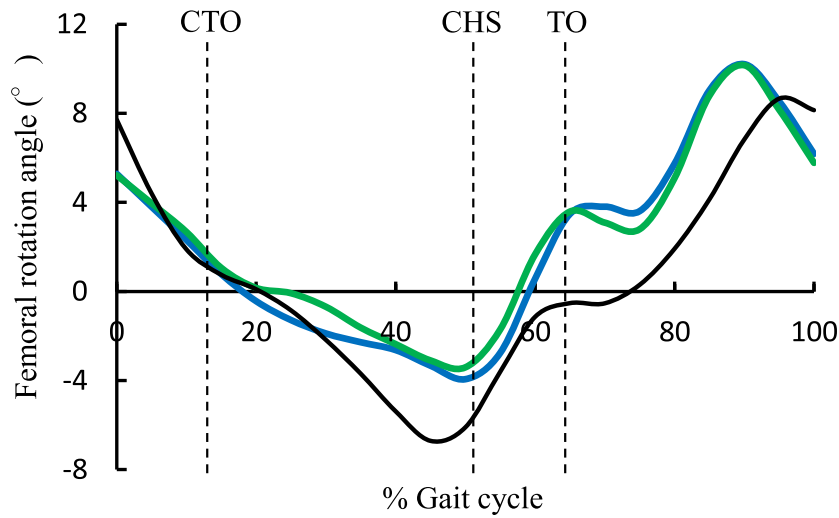

C Anterior $(+)$

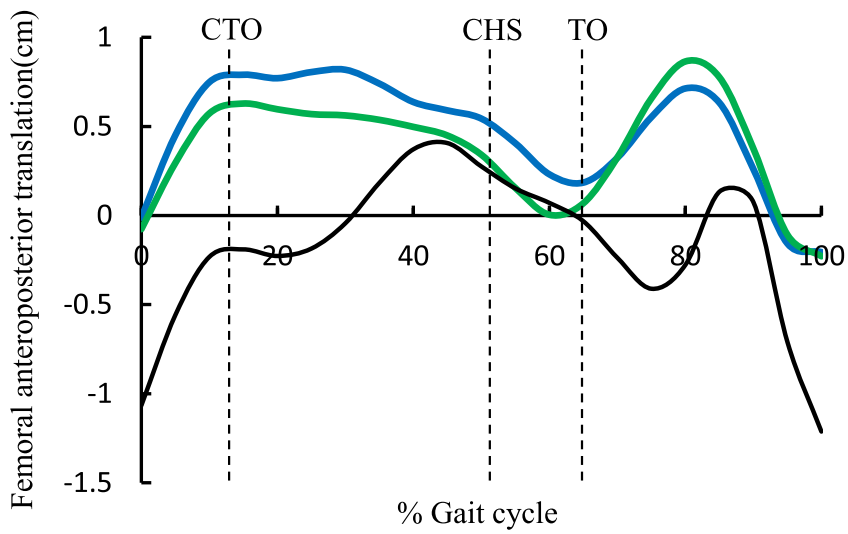

Fig. 2 Kinematics of the knee joint during walking. All three groups showed knee flexion twice during one walking cycle and external rotation in stance phase $(\mathbf{a}, \mathbf{b})$. In both the MP-CS and the MP-PS groups, the femur showed anterior translation during early stance phase, maintained the anterior position during mid-stance phase, and showed posterior translation during late stance phase to pre-swing phase (c). CTO, contralateral toe-off; CHS, contralateral heel strike; TO, toe-off; MP, medial pivot; CS, cruciate-substituting; PS, posterior-stabilized

surface shape of the MP-CS was preferred by patients when performing activities of daily living that induce medial pivot, such as standing up from a seated position, sitting down in a chair, and deep knee bending [15].
This study has several limitations. First, the participants in both MP groups were older than those in the control group and their walking speed was slower. The possibility that these limitations affected kinematics 


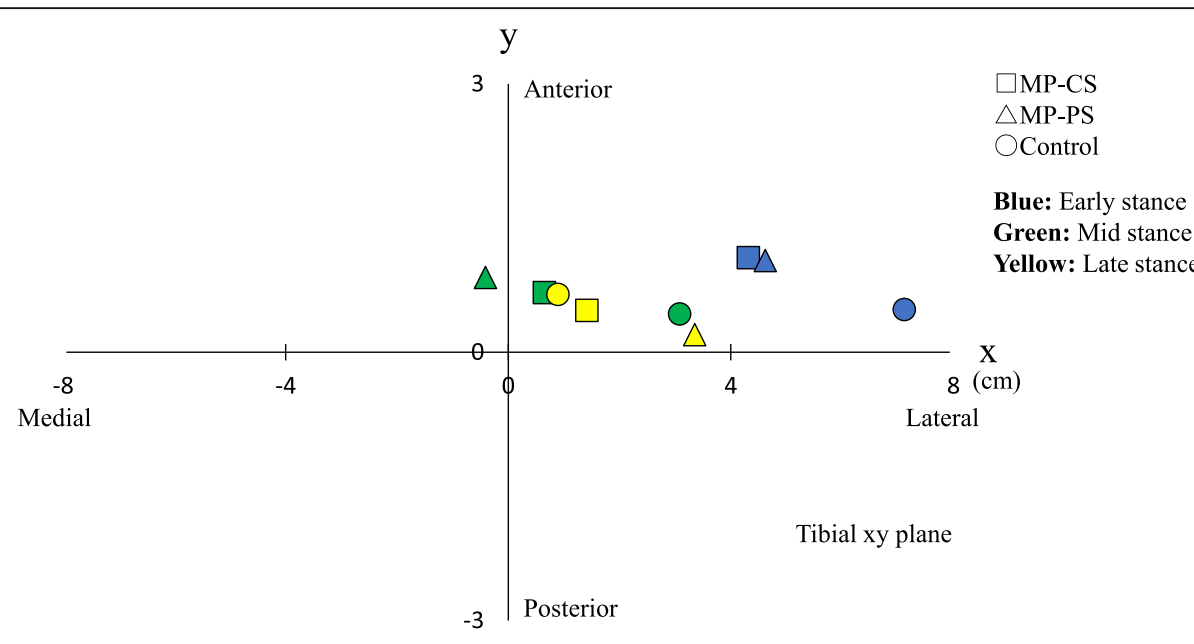

Fig. 3 Median COR during early stance, mid-stance, and late stance to swing phase of gait. Square, MP-CS group; triangle, MP-PS group; circle, control group. Blue indicates early stance, green indicates mid-stance, and yellow indicates late stance to pre-swing of gait. COR, center of axial rotation; MP, medial pivot; CS, cruciate-substituting; PS, posterior-stabilized

during walking cannot be excluded. Furthermore, patients with severe varus and valgus knee OA were excluded because the kinematics of knees with severe varus or severe valgus $\mathrm{OA}$ is significantly different from those of knees with general OA. Accordingly, if severe
OA-related deformity were concentrated in one group, it would likely be a confounding factor. Moreover, measurement error is a common problem with optical motion capture using skin markers. Alexander et al. [1] calculated the measurement error of the PC method

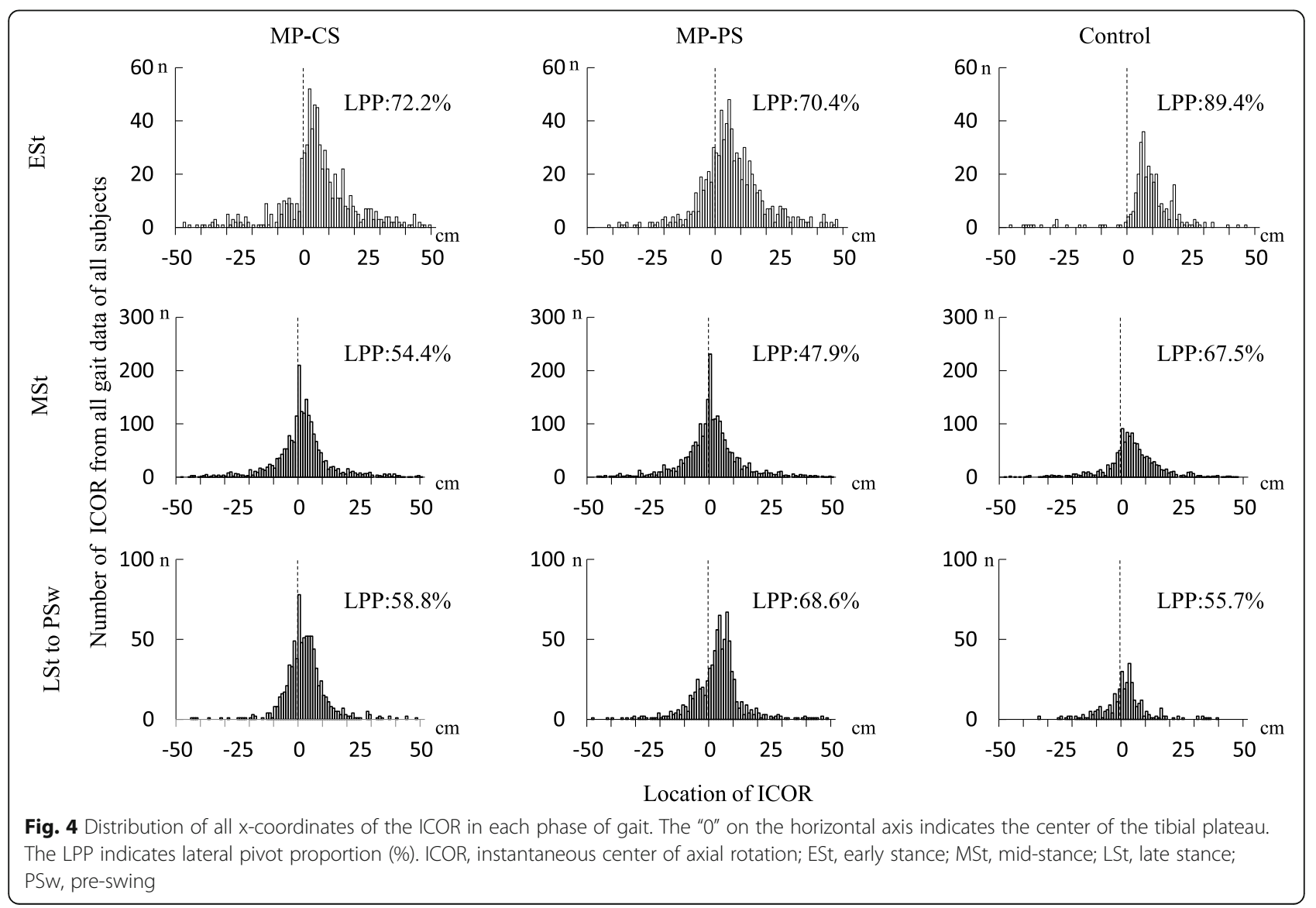



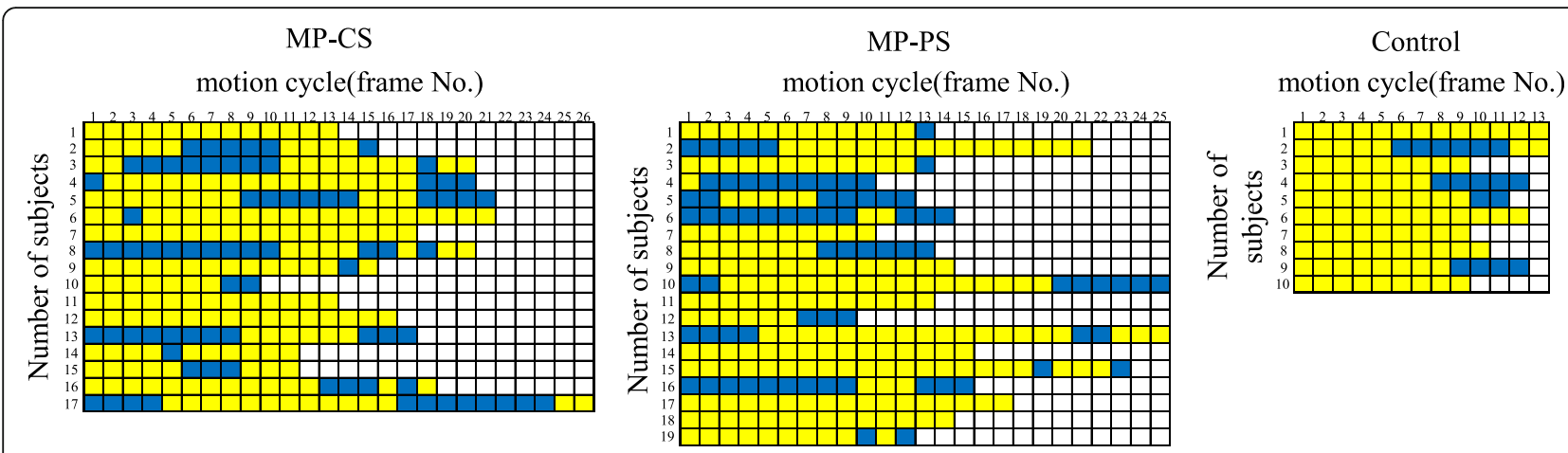

Fig. 5 Changes over time in location of the ICOR in early stance of gait. Blue indicates medial pivot and yellow indicates lateral pivot. The first frame number indicates heel strike and the last frame number indicates contralateral toe-off in each subject. ICOR, instantaneous center of axial rotation

using an external fixator device and reported that the maximum error was $4^{\circ}$ for rotation and $3 \mathrm{~mm}$ for translation, indicating the need for caution when interpreting changes smaller than these values. However, as mentioned previously, the findings for the kinematics of walking in healthy controls in our present study were qualitatively consistent with those of previous studies that used different methodologies [16-18]. This indicates that the PC method is suitable for analyzing walking.

In summary, this study found a small change in flexion and extension angles, anterior translation of the femur in early to mid-stance phases, and slight or no internal rotation of the tibia in the slight knee flexion position in stance phase during gait in knees that had undergone MP-CS or MP-PS. These findings resemble the compensatory motion observed in knees with ACL insufficiency. Moreover, MP-CS and MP-PS did not necessarily induce rotational motion centered on the medial ball-in-socket component during walking; rather, translational and lateral pivoting motions were observed. Therefore, the influence of MP surface geometry on kinematics during walking is thought to be limited.

\section{Conclusion}

In this study, the majority of patients who underwent medial pivot TKA showed the lateral pivot pattern during walking. For MP-CS, which is highly constrained in the medial ball-in-socket, it may be difficult to completely control tibiofemoral motion by geometry alone. Furthermore, our results do not dispel concerns about polyethylene wear and implant loosening during long-term follow-up. Postoperative satisfaction rated by KSS (2011) was significantly better in the MP-CS group than in the MP-PS group and was thought to reflect patients' preference for the surface geometry of the MP-CS when performing activities of daily living that cause medial pivot.

\section{Abbreviations}

3D: Three-dimensional; WBL ratio: Weight bearing line ratio; ACL: Anterior cruciate ligament; COR: Center of axial rotation; CS: Cruciate-substituting; FTA: Femorotibial angle; ICOR: Instantaneous center of axial rotation; KSS: Knee society score; MP: Medial pivot; PC: Point cluster; PCL: Posterior cruciate ligament; PS: Posterior-stabilized; PTEA: Projected transepicondylar axis; ROM: Range of motion; TEA: Transepicondylar axis; TKA: Total knee arthroplasty

\section{Acknowledgments}

This study was supported by Microport Orthopedics Inc. One author has received funding from Microport Orthopedics Inc.

\section{Authors' contributions}

KM contributed to the design of the research project, acquisition of data, analysis and interpretation of data, and drafting of the manuscript. YO contributed to design of the research project, overall supervision, and assistance with writing of the manuscript. TI and KU contributed to acquisition of the data and the analysis and interpretation of the data. KK and SS contributed to the software data analysis. KS and TM contributed to the analysis and interpretation of the data. All authors have read and approved the submitted manuscript.

\section{Funding}

This research was financially supported by MicroPort Orthopedics, Inc. (Arlington, VA).

\section{Availability of data and materials}

The datasets used and/or analyzed during the current study are available from the corresponding author on reasonable request.

\section{Ethics approval and consent to participate}

The study was approved by the ethics committee of the Suda Clinic institutional review board. All subjects provided written informed consent after receiving a detailed explanation of the study.

\section{Consent for publication}

Not applicable.

\section{Competing interests}

$\mathrm{YO}$ is an advisor to MicroPort Orthopedics, Zimmer Biomet, and Stryker Japan.

\section{Author details}

${ }^{1}$ Department of Rehabilitation, Hakodate Orthopedics Clinic, 2-115, Ishikawa-cho, Hakodate-shi, Hokkaido 041-0802, Japan. ²Department of Orthopedic Surgery, Hakodate Orthopedics Clinic, Hakodate, Japan. ${ }^{3}$ Department of Physical Therapy, Faculty of Health Sciences, Hokkaido University of Science, Sapporo, Japan. ${ }^{4}$ Department of Production Systems Eng., National Institute of Technology, Hakodate College, Hakodate, Japan. 
${ }^{5}$ Department of Complex and Intelligent Systems, Future University Hakodate, Hakodate, Japan.

Received: 7 July 2020 Accepted: 8 September 2020

Published online: 28 September 2020

\section{References}

1. Alexander EJ, Andriacchi TP (2001) Correcting for deformation in skin-based marker systems. J Biomech 34:355-361

2. Andriacchi TP, Alexander EJ, Toney MK, Dyrby C, Sum J (1998) A point cluster method for in vivo motion analysis: applied to a study of knee kinematics. J Biomech Eng 120:743-749

3. Banks SA, Hodge WA (2004) 2003 hap Paul award paper of the International Society for Technology in Arthroplasty: design and activity dependence of kinematics in fixed and mobile-bearing knee arthroplasties. J Arthroplast 19(7):809-816. https://doi.org/10.1016/j.arth.2004.04.011

4. Barnes CL, Blaha JD, DeBoer D, Stemniski P, Obert R, Carroll M (2012) Assessment of a medial pivot total knee arthroplasty design in a cadaveric knee extension test model. J Arthroplast 27(8):1460-1468. https://doi.org/10. 1016/j.arth.2012.02.008

5. Berchuck M, Andriacchi TP, Bach BR, Reider B (1990) Gait adaptations by patients who have a deficient anterior cruciate ligament. J Bone Joint Surg Am 72:871-877

6. Blaha JD, Mancinelli CA, Simons WH, Kish VL, Thyagarajan G (2003) Kinematics of the human knee using an open chain cadaver model. Clin Orthop 410:25-34. https://doi.org/10.1097/01.blo.0000063564.90853.ed

7. Dennis DA, Mahfouz MR, Komistek RD, Hoff W (2005) In vivo determination of normal and anterior cruciate ligament-deficient knee kinematics. J Biomech 38:241-253. https://doi.org/10.1016/j.jbiomech.2004.02.042

8. Fuentes A, Hagemeister N, Ranger P, Heron T, de Guise JA (2011) Gait adaptation in chronic anterior cruciate ligament-deficient patients: pivotshift avoidance gait. Clin Biomech 26:181-187. https://doi.org/10.1016/j. clinbiomech.2010.09.016

9. Galvin AL, Kang L, Udofia I, Jennings LM, McEwen HMJ, Jin Z, Fisher J (2009) Effect of conformity and contact stress on wear in fixed-bearing total knee prostheses. J Biomech 42:1898-1902. https://doi.org/10.1016/j.jbiomech. 2009.05.010

10. Gray HA, Guan S, Thomeer LT, Schache AG, de Steiger R, Pandy MG (2019) Three-dimensional motion of the knee-joint complex during normal walking revealed by mobile biplane x-ray imaging. J Orthop Res 37:615630. https://doi.org/10.1002/jor.24226

11. Grood ES, Suntay WJ (1983) A joint coordinate system for the clinical description of three-dimensional motions: application to the knee. J Biomech Eng 105:136-144

12. Ino T, Ohkoshi Y, Tatsunori M, Kawakami K, Suzuki S, Tohyama H (2015) Side-to-side differences of three-dimensional knee kinematics during walking by normal subjects. J Phys Ther Sci 27:1803-1807. https://doi.org/ https://doi.org/10.1589/jpts.27.1803

13. Iwaki H, Prinskerova V, Freeman MAR (2000) Tibiofemoral movement 1: the shapes and relative movements of the femur and tibia in the unloaded cadaver knee. J Bone Joint Surg Br 82-B:1189-1195

14. Karachalios T, Varitimidis S, Bargiotas K, Hantes M, Roidis N, Malizos KN (2016) An 11-to15-year clinical outcome study of the advance medial pivot total knee arthroplasty. Bone Joint J 98:1050-1055. https://doi.org/10.1302/ 0301-620X.98B8.36208

15. Komistek RD, Dennis DA, Mahfouz M (2003) In vivo fluoroscopic analysis of the normal human knee. Clin Orthop 410:69-81. https://doi.org/10.1097/01. blo.0000062384.79828.3b

16. Koo S, Andriacchi TP (2008) The knee joint center of rotation is predominantly on the lateral side during normal walking. J Biomech 41: 1269-1273. https://doi.org/10.1016/j.jbiomech.2008.01.013

17. Kozanek M, Hosseini A, Liu F, Van de Velde SK, Gill TJ, Rubash HE et al (2009) Tibiofemoral kinematics and condylar motion during the stance phase of gait. J Biomech 42(12):1877-1884. https://doi.org/10.1016/j. jbiomech.2009.05.003

18. Lafortune MA, Cavanagh PR, Sommer HJ, Kalenak A (1992) Threedimensional kinematics of the human knee during walking. J Biomech 25(4):347-357

19. Macheras GA, Galanakos SP, Lepetsos P, Anastasopoulos PP, Papadakis SA (2017) A long term clinical outcome of the medial pivot knee arthroplasty system. Knee 24:447-453. http://dx.doi.org/https://doi.org/10.1016/j.knee. 2017.01.008

20. McClelland AJ, Webster KE, Julian AF, Hylton BM (2011) Knee kinematics during walking at different speeds in people who have undergone total knee replacement. Knee 18:151-155. https://doi.org/10.1016/j.knee.2010.04.005

21. Miyazaki Y, Nakamura T, Kogame K, Saito M, Yamamoto K, Suguro T (2011) Analysis of the kinematics of total knee prostheses with a medial pivot design. J Arthroplast 26(7):1038-1044. https://doi.org/10.1016/j.arth.2010.08.015

22. Schmidt R, Komistek RD, Blaha JD, Penenberg BL, Maloney WJ (2003) Fluoroscopic analyses of cruciate-retaining and medial pivot knee implants. Clin Orthop 410:139-147. https://doi.org/10.1097/01.blo.0000063565.90853.a4

23. Warth LC, Ishmael MK, Deckard ER, Davis MZ, Meneghini RM (2017) Do medial pivot kinematics correlate with patient-reported outcomes after total knee arthroplasty? J Arthroplast 32:2411-2416. http://dx.doi.org/https://doi. org/10.1016/j.arth.2017.03.019

24. Zang, Q, Chen Z, Zhang J, Hu J, Peng Y, Fan X, Jin Z (2019) Insert conformity variation affects kinematics and wear performance of total knee replacements. Clin Biomech 65:19-25. https://doi.org/https://doi.org/10. 1016/j.clinbiomech.2019.03.016

\section{Publisher's Note}

Springer Nature remains neutral with regard to jurisdictional claims in published maps and institutional affiliations.

\section{Submit your manuscript to a SpringerOpen ${ }^{\circ}$ journal and benefit from:}

- Convenient online submission

- Rigorous peer review

- Open access: articles freely available online

- High visibility within the field

- Retaining the copyright to your article

Submit your next manuscript at $>$ springeropen.com 